\title{
Nonlinear Identification Method Corresponding to Muscle Property Variation in FES - Experiments in Paraplegic Patients
}

\author{
Mitsuhiro Hayashibe, Mourad Benoussaad, David Guiraud, Philippe Poignet, Charles Fattal
}

\begin{abstract}
A model-based Functional Electrical Stimulation (FES) would be very helpful for the adaptive movement synthesis of spinal-cord-injured patients. The nonlinearity of the neuromuscular system can be captured through modeling and identification process. However, there are still critical limitations in FES: rapid muscle fatigue and time-varying property. In actual FES, in order to minimize the fatigue, the intermittent stimulation is adopted. In this case, fatigue and recovery occur in sequence. Thus, the time-varying muscle response is really difficult to be predicted for FES force control. In this paper, we propose an identification method to identify unknown internal states and the maximal force parameter which are inside the nonlinear differential equation. Among the internal parameters of muscle model, maximal force Fm should be mainly changed corresponding to the current muscle condition. Muscle fatigue or recovery itself is difficult to be modeled and predicted, however observing the input-output information from the muscle, the adaptive estimation will be achieved to correspond to the varying muscle response effected by a fatigue or unknown metabolic factor of human system. This identification method itself can be expected to be applied for general use in rehabilitation robotics.
\end{abstract}

\section{INTRODUCTION}

The electrical stimulation to activate paralyzed musclelimb system and restore functional movements for subjects with Spinal Cord Injury (SCI) is termed Functional Electrical Stimulation (FES). FES has been succeeded to restore functional movements such as standing up and walking [1], [2] and it has been applied even in an implanted manner [3]. Although FES technology has already shown its great potential and advantage of improving mobility for SCI or post-stroke patient, it has not yet gained widespread clinical use due to difficulties, such as nonlinearity of the neuromuscular system, rapid muscle fatigue and inaccurate control of muscle forces [4], [5]. These issues severely limit FES for a prolonged use. The use of mathematical model would improve the FES control by using optimized operation for individual patients. A mathematical model may enable to describe the relevant characteristics of the patient's skeletal muscle and predict the precise force against certain stimulation. For the model-based control, identification is essential to realize better FES motion synthesis. In previous works, identification of FES-induced quadriceps dynamics was investigated in on-line [6],[7] , off-line [8], and blackbox modeling [9].

M. Hayashibe, M. Benoussaad, D. Guiraud, P. Poignet are with INRIA Sophia-Antipolis -DEMAR Project and LIRMM, UMR5506 CNRS UM2, 161 Rue Ada - 34392 Montpellier Cedex 5, France hayashibe, benoussaad, guiraudelirmm. fr

C. Fattal is with Propara Rehabilitation Center, 263 rue du Caducee, Parc Euromedecine - 34195 Montpellier Cedex 5, France
In order to have more physiologically oriented interpretation in FES muscle contraction, we prefer to use physiologically detailed muscle model. However, as the above identification work has been carried out using rather simplified muscle models, not many authors have suggested an extensive use of physiology based models which were designed also for the experimental identification. An adaptation of such model which has both microscopic and macrosopic view of muscle physiology in FES was reported in [10],[11]. Physiologically detailed modeling can lead a complexity of parameter identification in the nonlinear muscle dynamics. We proposed an experimental method to identify unknown internal states and parameters from the limited information. The identification of the nonlinear dynamics in stimulated skeletal muscle was presented for in-vivo rabbit test [12].

The nonlinearity of the neuromuscular system can be captured through modeling and identification process. However, we should remind that there are other critical limitations in FES: rapid muscle fatigue and time-varing property. The synchronous mode of stimulation in which all the muscle fibers are activated simultaneously causes fatigue to occur more rapidly in the activated paralyzed limb. A predictive fatigue modeling for FES was proposed [13] and fatigue time function was integrated in FES muscle modeling. Due to the differences in fatigability of motor units within a muscle, the force-stimulation intensity relationship may change with fatigue. As stated in [14], the understanding of the forcestimulation intensity relationship during fatigue is still on going study. Thus, numerical fatigue function can contribute to the reduction of model estimation error, however it is not enough for an accurate force estimation effected by fatigue. In actual FES, in order to minimize the fatigue, the intermittent stimulation is adopted. In this case, fatigue and recovery occur in sequence. The history-dependency of the muscle response to FES becomes significant. The fatigue in continuous stimulation is still easier to be predicted using evoked EMG, however recovery level is related to the subject's metabolic and electrolytic factors. Thus, the timevarying muscle response is really difficult to be predicted for FES force control. As an alternative solution, on-line adaptation can be considered. Ferrarin [15] applied adaptive algorithm to cope with muscle fatigue and muscle activation input was adjusted minimizing the modeling error.

In this paper, we propose an identification method for unknown internal states and the maximal force parameter in nonlinear multi-scale muscle model. Among the internal parameters, maximal force Fm should be mainly changed corresponding to the current muscle condition. Muscle fa- 
tigue or recovery itself is difficult to be modeled and predicted, however observing the input-output information from the muscle, the adaptive identification would be achived to correspond to the varying muscle response effected by a fatigue or unknown metabolic factor of human system.

This paper is organized as follows. In Sec. II, our multiscale physiological muscle model is described. In Sec. III, we formulate a nonlinear identification corresponding to muscle property changes in FES. We verify our approach in Sec. IV by demonstrating muscle force prediction by the identification using the latest response. Three palaplegic patients have been recruited and the cross validation shows the performance when the muscle response is largely effected by muscle fatigue.

\section{PHYSIOLOGICAL MUSCLE MODEL}

The mechanical representation of our model is based on a macroscopic Hill configuration [16]. Hence, its macroscopic property includes the property of so-called Hill-type model. However, its contraction dynamics of the contractile element is more detailed than Hill-type model and it includes also the dynamics of the microscopic muscle physiology. A with-mass mechanical model was used for the experimental identification of in-vivo rabbit skeletal muscle [12]. In human subjects, accurate force measurement directly from an isolated muscle is not accessible, thus simple Hill-Maxwell model is used as shown in Fig. 1. In this work, quadriceps muscle is investigated, then pennation angle was assumed to be negligible. The microscopic muscle physiology in our model is based on the two-state cross-bridge model of Huxley [17] and the distribution-moment model of Zahalak [18]. This model was originally proposed for FES control in [11], and it was also applied for EMG-force estimation in healthy subjects [19]. Here, it is summarized briefly.

\section{A. Sarcomere scale}

We assume that all the sarcomeres are identical and they contract and relax simultaneously so that the relationship between the two scales is proportional. If $S$ is the sarcomere length and $L_{c}$ is the length of contractile element, we can write $\left(S-S_{0}\right) / S_{0}=\left(L_{c}-L_{c 0}\right) / L_{c 0}=\varepsilon_{c}$ where $S_{0}, L_{c 0}$ are the ones at rest position.

Huxley proposed that a cross-bridge between actine filaments and myosine heads could exist in two biochemical states, attached and detached states. He postulated that one myosine head could attach to only one actine site. Then, the dynamics of the fraction $n(y, t)$ of the attached cross bridges is given by

$$
\frac{\partial n}{\partial t}+\frac{S_{0}}{h} \dot{\varepsilon}_{c} \frac{\partial n}{\partial y}=f(y, t)[1-n(y, t)]-g(y, t) n(y, t)
$$

Fig. 1. Macroscopic muscle-tendon model. where $h$ is the maximum elongation of the myosine spring, $y$ the normalized distance between actine binding site and myosine head. $n(y, t)$ is a distribution function representing the fraction of attached cross bridges relative to the normalized position $y . S_{0} \dot{\varepsilon}_{C}$ represents the velocity of the actine filament relative to the myosine filament. $f$ and $g$ denote the rate functions of attachment and detachment respectively.

The existence of attachment-detachment cycle is dependent on calcium concentration $\left[\mathrm{Ca}^{+2}\right]$ in the sarcomere as well as on the relative velocity between actin and myosin. When the velocity is high, the probability for crossbridges breaking is high. Thus, the chemical input $u(t)$, introduced by Bestel [20] that modifies the ability of the cross-bridges to attach or not, can be defined as follows:

$$
\begin{aligned}
& u(t)=\Pi_{c}(t) U_{c}+\left(1-\Pi_{c}(t)\right) U_{r} \\
& \Pi_{c}(t)=1 \text { during contraction, } 0 \text { else } \\
& (f+g)(y, t)=u(t)+\left|\dot{\varepsilon}_{c}\right|
\end{aligned}
$$

$U_{c}$ and $U_{r}$ are the level of chemical kinetics under contraction and relaxation phases respectively. Stiffness and force generated by a muscle sarcomere can be obtained by computing the first and second moment of the distribution $n(y, t)$.

\section{B. Myofiber and muscle unit scale}

The maximum available cross bridges could vary depending on the relative length of the contractile element. It is known as the force-length relationship $\left(f_{l}\left(\varepsilon_{c}\right)\right)$. Contrary to Hill model, we take into account this relation at the microscopic scale.

Let's define $k_{c}$ and $F_{c}$ as stiffness and force for the whole contractile element, and $N$ the number of all MUs. The number of recruited fibers is written as $\alpha N$ using recruitment ratio $\alpha$. Considering that each fiber is composed of identical sarcormeres in series, finally the desired model of contractile element is a set of differential equations as below:

$$
\begin{aligned}
& \dot{k}_{c}=-\left(u+\left|\dot{\varepsilon}_{c}\right|\right) k_{c}+\alpha k_{m} \Pi_{c}(t) U_{c} \\
& \dot{F}_{c}=-\left(u+\left|\dot{\varepsilon}_{c}\right|\right) F_{c}+\alpha F_{m} \Pi_{c}(t) U_{c}+k_{c} L_{c 0} \dot{\varepsilon}_{c}
\end{aligned}
$$

where $k_{m}=S_{0} N k_{0} f_{l}\left(\varepsilon_{c}\right) / L_{c 0}, F_{m}=N k_{0} h f_{l}\left(\varepsilon_{c}\right) / 2 . k_{0}$ $\left(\mathrm{Nm}^{-1}\right)$ is the maximum stiffness obtained when all the available bridges are attached in sarcomere scale.

\section{Computation of the whole dynamics}

The dynamics of contractile element coupled with the tendon in series should also meet the following equation:

$$
\dot{F}_{c}=k_{s}\left(L_{0} \dot{\varepsilon}-L_{c 0} \dot{\varepsilon}_{c}\right)
$$

where $k_{s}$ is the tendon stiffness, $L_{0}$ is the original length of musculotendinous formation and $\varepsilon$ its strain. In order to have one solution for two equations of $\dot{F}_{c}$, we must verify that: $k_{s} L_{c 0}+k_{c} L_{c 0}-F_{c}>0$. The differential of elongation $\varepsilon_{c}$ can be computed with the following equation:

$$
\dot{\varepsilon}_{c}=\frac{k_{s} L_{0} \dot{\varepsilon}+F_{c} u-\alpha F_{m} \Pi_{c}(t) U_{c}}{k_{s} L_{c 0}+k_{c} L_{c 0}-S_{\dot{\varepsilon}_{c}} F_{c}}
$$

$S_{\dot{\varepsilon}_{c}}$ is the sign of $\dot{\varepsilon}_{c}$. From the condition: $k_{s} L_{c 0}+k_{c} L_{c 0}-$ $F_{c}>0, S_{\dot{\varepsilon}_{c}}$ can be obtained from the sign of these terms: 
$k_{s} L_{0} \dot{\varepsilon}+F_{c} u-\alpha F_{m} \Pi_{c}(t) U_{c}$. Then we can compute $\dot{k}_{c}$ and $\dot{F}_{c}$ with (3). The internal state vector of this system should be set as $\mathbf{x}=\left[\begin{array}{lll}k_{c} & F_{c} & \varepsilon_{c}\end{array}\right]$.

\section{IDENTIFICATION METHOD}

\section{A. Probabilistic Inference in Dynamic State-Space Model}

The differential equations of skeletal muscle dynamics are straightly given in (3) and (5). The input controls of the model are the recruitment rate $\alpha$ and the chemical control input $u$. These two controls can be computed from FES input signal. The trigger of $u$ signal can be calculated by the timing of electrical stimulation considering the time delay caused by calcium dynamics. For the recruitment rate, it is defined by the current amplitude $I$ and Pulse Width (PW). In this work, we use same $I$ for all stimulations. Isometric knee torques at optimal knee position is measured at different PW. $\alpha$ is obtained from the normalized measured torques against maximum torque. In this case, $k_{c} F_{c} \varepsilon_{c}$ are timevarying states and only $F_{c}$ is accesible by the experimental measurement. $L_{c 0} k_{s} k_{m} F_{m}$ are unknown parameters. In this work, we use isometric force measurement for the identification of time-varying parameter in the model to detect the muscle property change. $L_{c 0} k_{s}$ are not timevarying parameters. These values were obtained from the literature [21]. $k_{m}$ is not significant parameter in the case of isometric contraction. Thus, $F_{m}$ is set as the unknown time-varying parameters to be estimated.

This model is a nonlinear state-space model, and statevariables are not measurable. Experimental data in human subject includes some noises. Therefore, we need a sequential probabilistic inference that estimates the hidden states of a dynamic system from a series of noisy measurements. When the model is highly non-linear, Extented KF may give particularly poor performance and an easy divergence. In skeletal muscle dynamics, its state-space is dramatically changed between contraction and relaxation phase. Then partial derivatives will be incorrect due to the discontinuity. Therefore, we adopted Sigma-Point Kalman Filter (SPKF) based on derivativeless statistical linearization. The initial idea was proposed by Julier [22], and well described by Merwe [23]. SPKF uses a deterministic sampling technique known as the unscented transform to pick a minimal set of sample points (called sigma points) around the mean. These sigma points are propagated through the true nonlinearity. This approach results in approximations that are accurate to at least the second order in Taylor series expansion. In contrast, EKF results only in first order accuracy.

The general Kalman framework involves estimation of the state of a discrete-time nonlinear dynamic system,

$$
\mathbf{x}_{k+1}=\mathbf{f}\left(\mathbf{x}_{k}, \mathbf{v}_{k}\right), \quad \mathbf{y}_{k}=\mathbf{h}\left(\mathbf{x}_{k}, \mathbf{n}_{k}\right)
$$

where $\mathbf{x}_{k}$ represents the internal state of the system to be estimated and $\mathbf{y}_{k}$ is the only observed signal. The process noise $\mathbf{v}_{k}$ drives the dynamic system, and the observation noise is given by $\mathbf{n}_{k}$. The filter starts by augmenting the state vector to $\mathrm{L}$ dimensions, where $\mathrm{L}$ is the sum of dimensions in the original state, model noise and measurement noise. The corresponding covariance matrix is similarly augmented to a $\mathrm{L}$ by $\mathrm{L}$ matrix. In this form, the augmented state vector $\hat{\mathbf{x}}_{k}^{a}$ and covariance matrix $\mathbf{P}_{k}^{a}$ can be defined as in (7)(8).

$$
\begin{aligned}
& \hat{\mathbf{x}}_{k}^{a}=E\left[\mathbf{x}_{k}^{a}\right]=\left[\begin{array}{lll}
\hat{\mathbf{x}}_{k}^{T} & \overline{\mathbf{v}}_{k}^{T} & \overline{\mathbf{n}}_{k}^{T}
\end{array}\right]^{T} \\
& \mathbf{P}_{k}^{a}=E\left[\left(\mathbf{x}_{k}^{a}-\hat{\mathbf{x}}_{k}^{a}\right)\left(\mathbf{x}_{k}^{a}-\hat{\mathbf{x}}_{k}^{a}\right)^{T}\right] \\
& =\left[\begin{array}{ccc}
\mathbf{P}_{\mathbf{x}_{k}} & 0 & 0 \\
0 & \mathbf{R}_{\mathbf{v}_{k}} & 0 \\
0 & 0 & \mathbf{R}_{\mathbf{n}_{k}}
\end{array}\right]
\end{aligned}
$$

where $\mathbf{P}_{\mathbf{x}}$ is the state covariance, $\mathbf{R}_{\mathbf{v}}$ is the process noise covariance, $\mathbf{R}_{\mathbf{n}}$ is the observation noise covariance.

In the process update, the $2 \mathrm{~L}+1$ sigma points are computed based on a square root decomposition of the prior covariance as in (9), where $\gamma$ is scaling parameter that determines the spread of the sigma-points around prior mean. The augmented sigma point matrix is formed by the concatenation of the state sigma point matrix, the process noise sigma point matrix, and the measurement noise sigma point matrix, such that $\mathcal{X}^{a}=\left[\begin{array}{lll}\left(\mathcal{X}^{x}\right)^{T} & \left(\mathcal{X}^{v}\right)^{T} & \left(\mathcal{X}^{n}\right)^{T}\end{array}\right]^{T} \cdot \omega_{i}^{c}$ and $\omega_{i}^{m}$ are the sigma point weights.

$$
\mathcal{X}_{k-1}^{a}=\left[\begin{array}{lll}
\hat{\mathbf{x}}_{k-1}^{a} & \hat{\mathbf{x}}_{k-1}^{a}+\gamma \sqrt{\mathbf{P}_{k-1}^{a}} & \hat{\mathbf{x}}_{k-1}^{a}-\gamma \sqrt{\mathbf{P}_{k-1}^{a}}
\end{array}\right]
$$

Predicted mean and covariance are computed as in (11)(12) and predicted observation is calculated like (14).

$$
\begin{aligned}
\mathcal{X}_{k \mid k-1}^{x} & =\mathbf{f}\left(\mathcal{X}_{k-1}^{x}, \mathcal{X}_{k-1}^{v}\right) \\
\hat{\mathbf{x}}_{k}^{-} & =\sum_{i=0}^{2 L} \omega_{i}^{m} \mathcal{X}_{i, k \mid k-1}^{x} \\
\mathbf{P}_{\mathbf{x}_{k}}^{-} & =\sum_{i=0}^{2 L} \omega_{i}^{c}\left(\mathcal{X}_{i, k \mid k-1}^{x}-\hat{\mathbf{x}}_{k}^{-}\right)\left(\mathcal{X}_{i, k \mid k-1}^{x}-\hat{\mathbf{x}}_{k}^{-}\right)^{T} \\
\mathcal{Y}_{k \mid k-1} & =\mathbf{h}\left(\mathcal{X}_{k \mid k-1}^{x}, \mathcal{X}_{k-1}^{n}\right) \\
\hat{\mathbf{y}}_{k}^{-} & =\sum_{i=0}^{2 L} \omega_{i}^{m} \mathcal{Y}_{i, k \mid k-1}
\end{aligned}
$$

The predictions are then updated with new measurements by first calculating the measurement covariance and statemeasurement cross correlation matrices, which are then used to determine the Kalman gain. Finally, updated estimate and covariance are decided through this kalman gain as below.

$$
\begin{aligned}
\mathbf{P}_{\tilde{\mathbf{y}}_{k}} & =\sum_{i=0}^{2 L} \omega_{i}^{c}\left(\mathcal{Y}_{i, k \mid k-1}-\hat{\mathbf{y}}_{k}^{-}\right)\left(\mathcal{Y}_{i, k \mid k-1}-\hat{\mathbf{y}}_{k}^{-}\right)^{T} \\
\mathbf{P}_{\mathbf{x}_{k} \mathbf{y}_{k}} & =\sum_{i=0}^{2 L} \omega_{i}^{c}\left(\mathcal{X}_{i, k \mid k-1}^{x}-\hat{\mathbf{x}}_{k}^{-}\right)\left(\mathcal{Y}_{i, k \mid k-1}-\hat{\mathbf{y}}_{k}^{-}\right)^{T} \\
\hat{\mathbf{x}}_{k} & =\hat{\mathbf{x}}_{k}^{-}+\mathbf{K}_{k}\left(\mathbf{y}_{k}-\hat{\mathbf{y}}_{k}^{-}\right), \quad \mathbf{K}_{k}=\mathbf{P}_{\mathbf{x}_{k} \mathbf{y}_{k}} \mathbf{P}_{\tilde{\mathbf{y}}_{k}}^{-1} \\
\mathbf{P}_{\mathbf{x}_{k}} & =\mathbf{P}_{\mathbf{x}_{k}}^{-}-\mathbf{K}_{k} \mathbf{P}_{\tilde{\mathbf{y}}_{k}} \mathbf{K}_{k}^{T}
\end{aligned}
$$

These process update and measurement update should be recursively calculated in $k=1, \ldots, \infty$ until the end point of the measurement. For the implementation, we used efficient square-root forms which propagate the square-root of the state covariance directly in Cholesky factored form. 


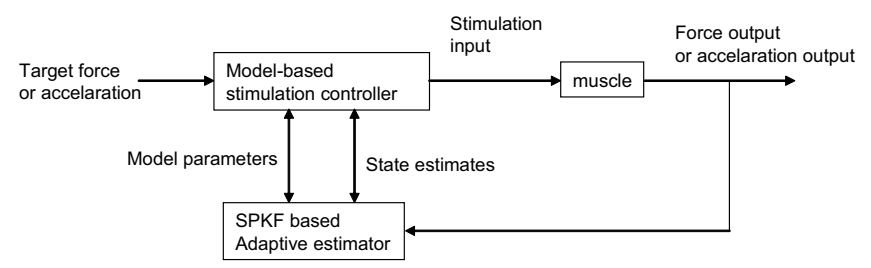

Fig. 2. Concept of adaptive estimation for muscle property change.

\section{B. State and Parameter estimater}

A concept of adaptive estimation for muscle property changes is shown in Fig. 2. The future stimulation controller for FES would need accurate estimates of the system states as well as parameters in order to robustly control the paralyzed muscle corresponding to its time-varying property. In this paper, according to this concept, we try to verify the feasibility of SPKF based adaptive estimator to realize state and parameter estimation in nonlinear dynamics which has multi-scale muscle physiology.

At first, estimation test was made to check the model observability using noise added simulation data for online computation to detect the internal parameter changes. Ramdom gaussian noise added force was prepared by the simulation. As in Fig. 3, output force sequence which has similar intensity was generated, however internal parameter $F_{m}$ was changed for each stimulation corresponding to muscle property change. The muscle force sequence was filtered through SPKF based estimator continuously and state $\epsilon_{c}$ and parameter $F_{m}$ were estimated. State $\epsilon_{c}$ was successfully obtained as you can see its true value in Fig. 4 and The change of $F_{m}$ also could be detected accurately only from the muscle force observation updates.

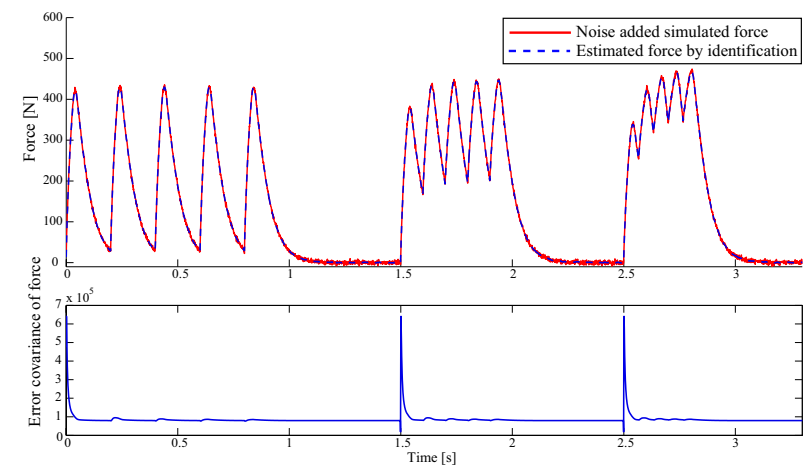

Fig. 3. Estimation test to check the model observability using noise added simulation data to detect the internal parameter changes. Red solid line: noise added simulation force, blue dash line: estimated force by identification. The figure in bottom shows the error covariance.

\section{EXPERIMENTAL RESULTS}

\section{A. Experimental Setup}

Experiments were conducted on three male subjects, who have complete paralysis ASIA A of lower extremity. An authorization from the ethical committee and an agreement from each subject were obtained. The general information about the subjects is summarized at Table I. The paraplegic patients were seated on a chair with their hip flexed at approximately $90^{\circ}$ and their shank fixed on BIODEX system.
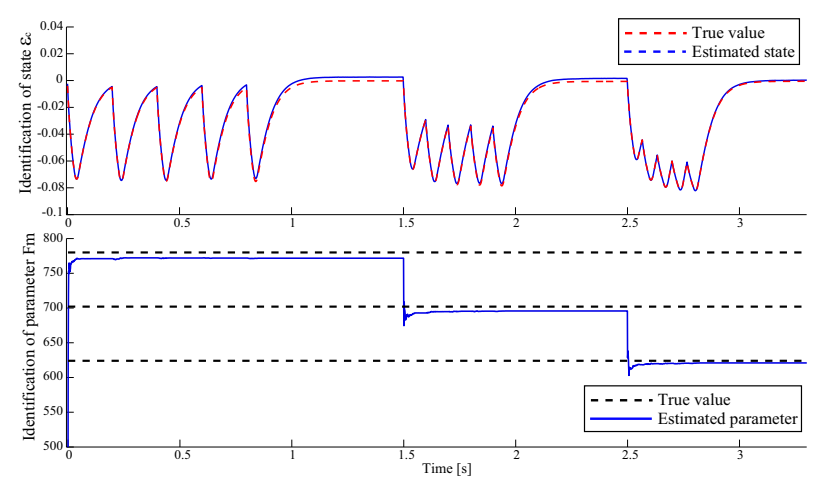

Fig. 4. Top: estimated result for state $\epsilon_{c}$. Red dash line shows true value and blue line shows estimated state in the identification. Bottom: estimated parameter $F_{m} . F_{m}$ was changed for each stimulation corresponding to muscle property change. The change of $F_{m}$ could be detected accurately only from the muscle force in Fig. 3.

The joint torques around knee were recorded in isometric condition with $2 \mathrm{KHz}$ sampling frequency through the dynamometer as in Fig. 5. The quadriceps muscle group was stimulated through surface electrodes for the knee extension. The specific fixed amplitude $I$ was chosen previously for each subject. During the experiments, the pulse width $P W$ was modulated to have different level of muscle response. The applied stimulation signal and the EMG $(4 \mathrm{KHz}$ sampling frequency) were recorded through BIOPAC MP100 acquisition system.

The measured torque was converted to force scale devided by the moment arm which was obtained from the Hawkins equation of muscle length at different joint angles [24] combined with the anthropometrical estimations of the limbs length [25] for each subject. The recruitment curve for $\alpha$ was obtained at the begining of the experiment by modulating PW. In the custom of FES, once PW modulation is chosen as control input, fixed frequency is applied during the course of stimulation. The difference of frequency changes temporal summation in muscle contraction. Normally, we used $20 \mathrm{~Hz}$ before tetanic frequency to minimize fatigue. However, when we check model reproduction ability, $30 \mathrm{~Hz}$ and $15 \mathrm{~Hz}$ were applied for tetanic contraction and unfused contraction respectively.

TABLE I

PATIENTS CHARACTERISTICS

\begin{tabular}{l|l|l|l|l|l}
\hline Subject & $\begin{array}{l}\text { Age } \\
\text { (years) }\end{array}$ & $\begin{array}{l}\text { Weight } \\
(\mathrm{Kg})\end{array}$ & $\begin{array}{l}\text { Height } \\
(\mathrm{m})\end{array}$ & $\begin{array}{l}\text { Injury } \\
\text { level }\end{array}$ & $\begin{array}{l}\text { Post injury } \\
\text { (years) }\end{array}$ \\
\hline \hline BP (S1) & 46 & 85.6 & 1.75 & T4 & 14 \\
\hline LT (S2) & 37 & 72 & 1.75 & T6 & 12 \\
\hline BD (S3) & 46 & 94 & 1.88 & T10 & 14 \\
\hline
\end{tabular}

\section{B. Direct Validation}

At first, to verify our identification method using the inputoutput relationship from experimental measurement in SCI patients, direct validation was made to see its identification performance for the multi-scale physiological muscle model. Muscle force responses in one paraplegic patient (S1) are given in Fig. 6 respectively in $15 \mathrm{~Hz}$ and $20 \mathrm{~Hz}$ stimulation frequency. The identification was executed by SPKF estimator to obtain parameter $F_{m}$ with different initial values as 


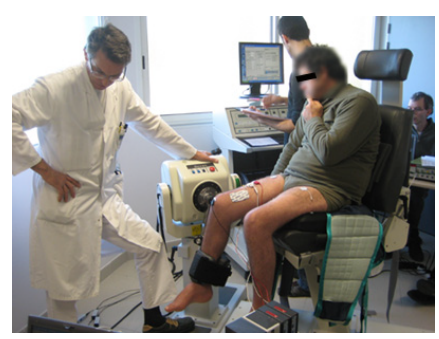

Fig. 5. The experimental setup: the torques around knee joint are measured in isometric condition through the Biodex system.

in Fig. 7. The stable identification result could be obtained with every initial value in SPKF. The estimation with the identified model can be confirmed with the measured force as direct validation in Fig. 8. The normalized root mean squared deviation (NRMSD) between the model estimations and measured values in force plateau was 4.87 percent in average.

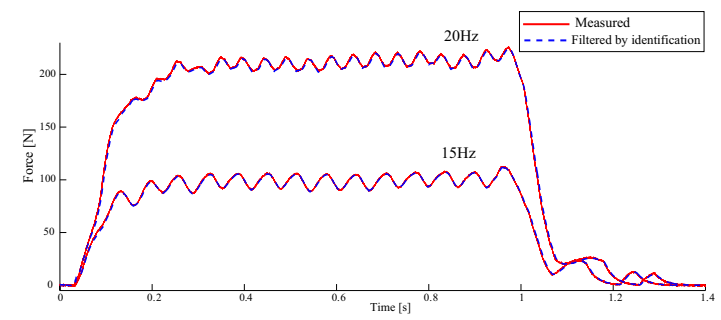

Fig. 6. Muscle force response in paraplegic patient in $15 \mathrm{~Hz}$ and $20 \mathrm{~Hz}$ and its identification by SPKF.

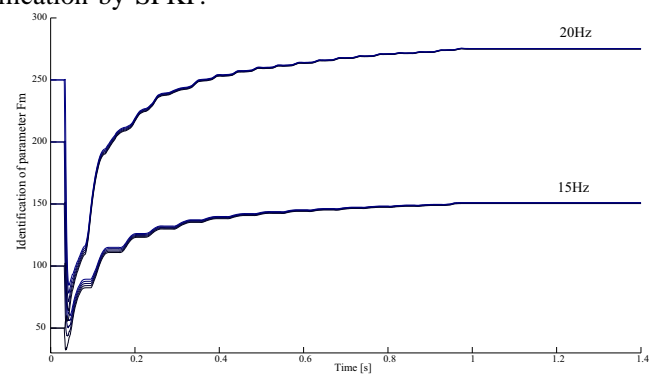

Fig. 7. Identification of parameter $F_{m}$ with different initial values.

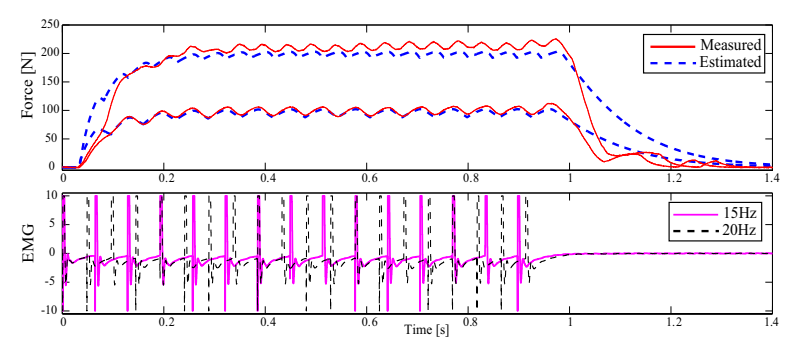

Fig. 8. Direct validation: measured force and model estimation.

\section{Cross Validation}

Next, we investigated the measured data in another subject (S2) for cross validation to see its model-based predictive performance. As described in the introduction, major critical limitations in FES control are rapid muscle fatigue and timevaring property. The synchronous mode of stimulation in which all the muscle fibers are activated simultaneously causes fatigue to occur more rapidly. Even if we try to use the intermittent stimulation, it is really difficult to capture and predict the muscle force effected by fatigue and recovery. This history-dependency of the muscle response is also subject-specific and daily changing. During the course of our experimental protocol, we found that the muscle response is in fact different even with same stimulation parameter as in Fig. 9. In this subject, the muscle response varied with 45 percent in maximum with the same stimulation. How to modulate $\mathrm{PW}$ is actually different for each stimulation protocol, however the force plateau should be same level if there is no muscle property change. This case was especially significant, the other averaged subject also showed the force decrease of 26 percent with the same experimental protocol.

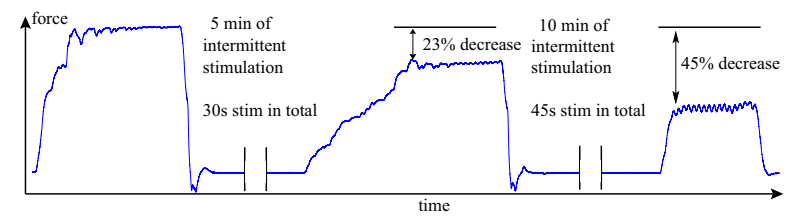

Fig. 9. Example of muscle force transition effected by fatigue with similar condition of stimulation ( $\mathrm{PW}=300 \mu \mathrm{S}, 20 \mathrm{~Hz})$.

However, we also found that muscle fatigue appears basically as long-term characteristics change not as sudden force drop. Therefore, we tried to identify the model using the input-output relationship in the temporal vicinity of the target timing. Simply if we re-identify the model using the last input-output relationship then the prediction with the recently identified model would be possible to minimize the effect of the time-varying muscle property. For each stimulation frequence of $15 \mathrm{~Hz}, 20 \mathrm{~Hz}, 30 \mathrm{~Hz}$, the input-output data in $300 \mu S$ was identified to obtain latest parameter of $F_{m}$ and the identified model was applied to estimate the muscle response in $250 \mu S$ and $150 \mu S$ as cross validation. These different PW stimulations were carried out with the interval of 1.5 minutes. Cross validation result can be seen as in Fig. 10-12. Normalized RMS deviations for the cross validation are shown in Table II. If we remind muscle response is really difficult to be predicted due to fatigue and also metabolic factors for each subject, this result suggests that the activated force prediction can be performed by adaptive identification even when the muscle response is largely effected by a muscle fatigue in FES. As same as first 2 subjects, the muscle response was largely varied in S3 subject, however similar quality of prediction could be performed.

TABLE II

NORMALIZED RMS DEVIATIONS FOR CROSS VALIDATION

\begin{tabular}{l|l|l|l}
\hline Frequency & $P W 300 \mu s$ & $P W 250 \mu s$ & $P W 150 \mu s$ \\
\hline \hline $15 \mathrm{~Hz}$ & 4.93 & 4.76 & 7.96 \\
\hline $20 \mathrm{~Hz}$ & 4.20 & 9.49 & 8.15 \\
\hline $30 \mathrm{~Hz}$ & 6.87 & 8.93 & 2.47 \\
\hline
\end{tabular}

\section{CONCLUSIONS}

Nonlinear identification method corresponding to muscle property change was proposed for FES control. Experimental validation tests in SCI patients were carried out to confirm 


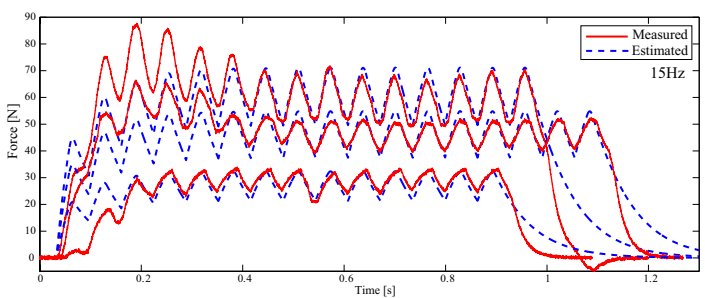

Fig. 10. Cross validation: measured force and model estimation in PW of $300 \mu S, 250 \mu S$ and $150 \mu S$ and in $15 \mathrm{~Hz}$. The input-output data in $300 \mu S$ (largest one) was identified and the identified model was applied to estimate the muscle response in $250 \mu S$ and $150 \mu S$.

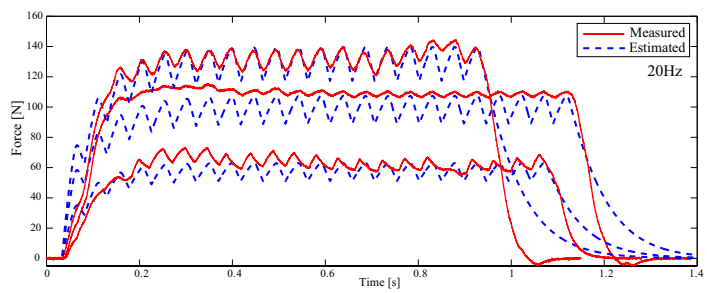

Fig. 11. Cross validation: measured force and model estimation in PW of $300 \mu S, 250 \mu S$ and $150 \mu S$ and in $20 \mathrm{~Hz}$.

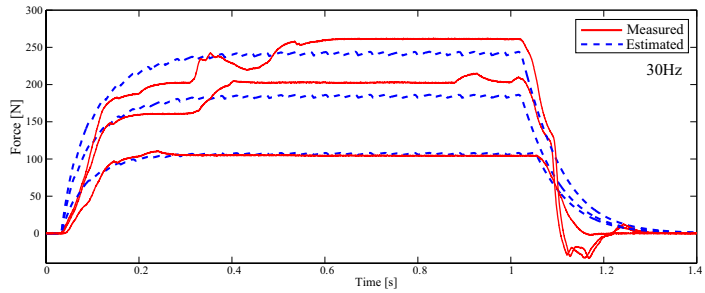

Fig. 12. Cross validation: measured force and model estimation in PW of $300 \mu S, 250 \mu S$ and $150 \mu S$ and in $30 \mathrm{~Hz}$.

the feasibility of the proposed method. Muscle response was changed in time when fatigue was appeared fast in FES. However, it can be feasible solution to make adaptive estimation for internal parameter and to correspond to the varying muscle response effected by a fatigue or unknown metabolic factor of human system. Cross validation showed the performance of force prediction even if the muscle response is largely effected by a muscle fatigue in FES. Some predictive errors are still remained as in Table II, however it can be expected to be cancelled by feedback controller. Even in the use of feedback control, it is still important to assess the muscle fatigue condition for the patient in the use of FES. Statistical analysis with more results is planned for future work.

\section{ACKNOWLEDGMENTS}

The authors gratefully acknowledge P. Benoit, R. Passama and M. Papaiordanidou for their assistance in experiments.

\section{REFERENCES}

[1] A. Kralj, T. Bajd. "Functional Electrical Stimulation: Standing and Walking After Spinal Cord Injury". Boca Raton: CRC Press Inc. 1989.

[2] R. Kobetic, R.J. Triolo, E.B. Marsolais, "Muscle Selection and Walking Performance of Multichannel FES Systems for Ambulation in Paraplegia",IEEE Trans. On Rehabilitation Engineering, Vol 5, N1, p23-29, 1997.
[3] D. Guiraud, T. Stieglitz, K.P. Koch, J.L. Divoux, P. Rabischong, ”An implantable neuroprosthesis for standing and walking in paraplegia: 5-year patient follow-up", J. Neural Eng., vol. 3, pp 268-275, 2006.

[4] Durfee K.W. "Control of standing and gait using electrical stimulation influence of muscle model complexity on control strategy", Prog. Brain Res., 97, 369-381, 1993.

[5] R. Riener "Model-based development of neuroprostheses for paraplegic patients", The Royal Society, 354, pp. 877-894, 1999.

[6] H. J. Chizeck, S. Chang, R. B. Stein, A. Scheiner, D. C. Ferencz, "Identification of Electrically Stimulated Quadriceps Muscles in Paraplegic Subjects", IEEE Trans. on Biomedical Engineering, vol. 46, pp 51-61,1999.

[7] T. Schauer, N.-O. Negard, F. Previdi, K. J. Hunt, M. H. Fraser, E Ferchland, J. Raisch, "Online identification and nonlinear control of the electrically stimulated quadriceps muscle", Control Engineering Practice, vol. 13, pp 1207-1219, 2004

[8] H. M. Franken, P. H. Veltink, R. Tijsmans, G.H. Nijmeijer and H.B. Boom, "Identification of quadriceps-shank dynamics using randomized interpulse interval stimulation", IEEE Trans. on Rehabilitation Engineering, vol. 3, no 2, pp 182-192, 1995.

[9] F. Previdi, "Identification of black-box nonlinear models for lower limb movement control using functional electrical stimulation", Control Engineering Practice, vol. 10, pp 91-99, 2002.

[10] R. Riener, J. Quintern, E. Psaier, G. Schmidt, "Physiological based multi-input model of muscle activation", Neuroprosthetics From Basic Research To Clinical Applications, Springer-Verlag, vol. 12, pp 95114, 1996.

[11] H. El Makssoud, D. Guiraud, P. Poignet, Mathematical muscle model for Functional Electrical Stimulation control strategies. IEEE/Int. Conf. on Robotics and Automation, pp. 1282-1287, 2004.

[12] M. Hayashibe, P. Poignet, D. Guiraud, H. El Makssoud, "Nonlinear identification of skeletal muscle dynamics with Sigma-Point Kalman Filter for model-based FES", IEEE/Int. Conf. on Robotics and Automation, pp. 2049-2054, 2008.

[13] J. Ding, A. S. Wexler, S. A. Binder-Macleod, "Mathematical models for fatigue minimization during functional electrical stimulation", Journal of Electromyography and Kinesiology, vol. 13, pp 575-588, 2003.

[14] L. Chou, S. A. Binder-Macleod, "The Effects Of Stimulation Frequency And Fatigue On The Force-Intensity Relationship For Human Skeletal Muscle”, Clin. Neurophysiol. vol. 118, no 6, pp 1387-1396, 2007.

[15] M. Ferrarin, F. Palazzo, R. Riener, J. Quintern, "Model-Based Control of FES-Induced Single Joint Movements", IEEE Trans. on Rehabilitation Engineering, vol. 9, no 3, pp 245-257, 2001.

[16] F.E. Zajac "Muscle and tendon: properties, models, scaling and application to biomechanics and motor control". CRC Critic. Rev. in Biomed. Eng. 17: 359-411, 1989.

[17] A. F. Huxley, Muscle structure and theories of contraction. Progress in Biophysics and Biophysical Chemistry, vol. 7, pp. 255-318, 1957.

[18] G. I. Zahalak, A distribution-moment approximation for kinetic theories of muscular contraction. Mathematical Biosciences, vol. 55, pp. 89-114, 1981

[19] M. Hayashibe, D. Guiraud, P. Poignet, "EMG-to-force estimation with full-scale physiology based muscle model", IEEE/RSJ/Int. Conf. on Intelligent RObots and Systems, St. Louis, MO, USA, 2009.

[20] J. Bestel, M. Sorine, A differential model of muscle contraction and applications. In schloessmann Seminar on Mathematical Models in Biology, Chemistry and Physics, Max Plank Society, Bad Lausick, Germany, May 19-23, 2000

[21] S.L. Delp, "Surgery simulation: a computer graphics system to analyze and design musculoskeletal reconstructions of the lower limb", Dissertation, Stanford University, CA, USA, 1990.

[22] S.J. Julier, J.K. Uhlmann, "A New Extension of the Kalman Filter to Nonlinear Systems". In Proc. of AeroSence: 11th Int. Symp. on Aerospace.Defence Sensing, Simulation and Controls ,1997.

[23] R. Merwe, E. Wan, "Sigma-Point Kalman Filters for Probabilistic Inference in Dynamic State-Space Models". Workshop on Advances in Machine Learning, Montreal, June 2003.

[24] D. Hawkins and M. Hull, "A method for determining lower extremity muscle-tendon lengths during flexion/extension movements", Journal of Biomechanics, vol. 23, pp. 487-494, 1990.

[25] P. de Leva, "Adjustments to zatsiorsky-seluyanov as segment inertia parameters", Journal of Biomechanics, vol. 29, pp. 1223-1230, 1996. 